\title{
DESIGN OF OBSTACLE DETECTION AND AVOIDANCE SYSTEM FOR GUANAY II AUV *
}

\author{
C. Galarza, I. Masmitja, J. Prat and S. Gomàriz
}

\begin{abstract}
This paper presents the design of an obstacle avoidance algorithm for Guanay II AUV. The obstacle detection system disposes a SONAR and its use guarantees the safety in navigation of $A U V$. Obstacle avoidance is performed based on a fuzzy reactive architecture for different forward speeds of the vehicle. The simulation results obtained through the implementation of the algorithms designed in Matlab validate the designs.
\end{abstract}

\section{INTRODUCTION}

Guanay II [1] (Fig. 1) is an underwater autonomous vehicle developed by SARTI group of the Polytechnic University of Catalonia, with the objective of providing a platform for measuring different oceanographic variables such as temperature and salinity of a water column, with high spatial and temporal resolution simultaneously.

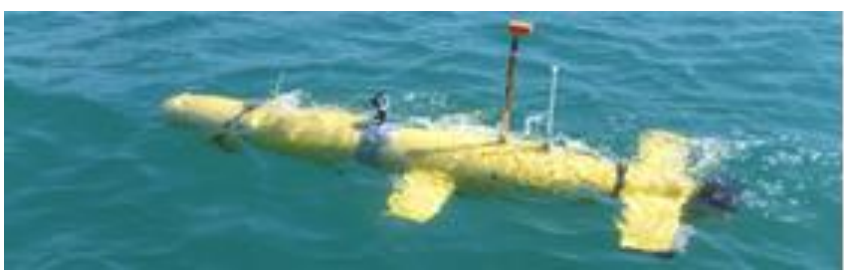

Figure 1. Guanay II AUV

The control system implemented in the Guanay II allows the vehicle to follow a predetermined navigation trajectory, marked by waypoints. At work [2] J. González et al. obtained the hydrodynamic model of the vehicle Guanay II with 3 Degrees of Freedom (DOF): surge, sway and yaw. This model allowed the design of the navigation control, composed by two loops. An outer loop (trajectory control), that carry out the tracking of the route pre-established navigation, based on strategies Pure-pursuit and Path Following and an inner loop that regulates the forward speed of the vehicle and the yaw angle.

The initial design of this AUV did not endowed of the vehicle with instrumentation or any obstacle detection system, and nor was it considered any obstacle avoidance system, objective of work that presented.

*Research supported by SARTI Research Group. Electronics Dept. Universitat Politècnica de Catalunya (UPC). Rambla Exposició 24, 08800,

Vilanova i la Geltrú. Barcelona. Spain. (+34) 938967200.

C.Galarza; e-mail: cesar.mauricio.galarza@estudiant.upc.edu.

I.Masmitja; e-mail: ivan.masmitja@upc.edu

J.Prat; e-mail: jordi.prat@upc.edu

S.Gomàriz; e-mail: spartacus.gomariz@upc.edu

\section{OBSTACle Detection System For Guanay II AUV}

Guanay II AUV [1] [2], is a vehicle designed to navigate on the surface and make vertical profiles. Its dynamic characteristics allow a maximum speed of navigation of $1 \mathrm{~m} / \mathrm{s}$ and a turning radius that varies with the speed according to Fig. 2.

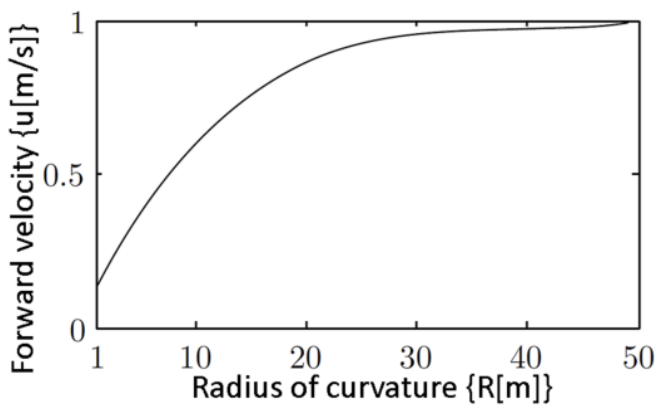

Figure 2. Maximum forward velocity $u$ regarding the radius of curvature [2]

Previous work [3], an obstacle detection system was designed incorporating to the vehicle a SONAR MK3 [4]. This SONAR detects the existence of obstacles in the trajectory of navigation and establishes its location and distance to the vehicle. This is achieved by analyzing the area scanned by the SONAR (Fig. 3). This area is divided into 3 sectors (right, center and left) each of $30^{\circ}$. For each of the sectors, the minimum distance between the vehicle and the obstacle is determined.

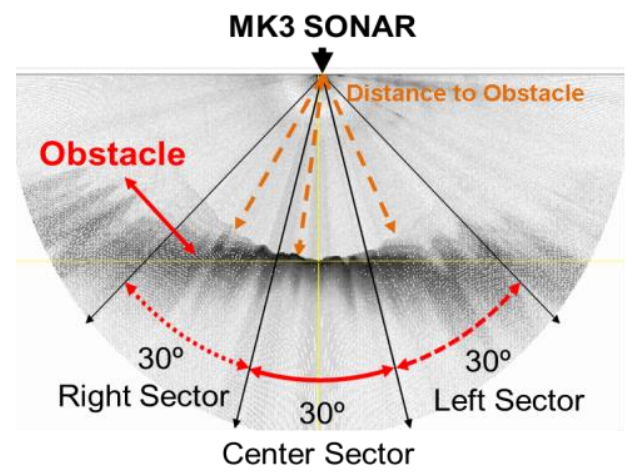

Figure 3. Description of the functioning of obstacle detection system [3]

III. Collision AVOIDANCE SyStems “CAS” (Literature REVIEW)

According to the literature consulted, some authors like Øystein [5] and Seto [6] pose different obstacle avoidance 
strategies that can be classified into: reactive, deliberative and hybrid. These strategies include the use of a sensor type SONAR to recognize or detect obstacles, and are executed in parallel to the trajectory control.

Reactive architecture is based on a structure of flow of sensor and action. A SONAR is used to perceive the environment and activate specific behaviors based on predefined rules in the presence of an obstacle. The performance of this architecture is often excellent when it comes to sudden obstacles and unknown environments, because of its short reaction time and robustness.

In this type of architecture, the avoidance system interacts with the trajectory control system when an obstacle is detected in the route of the vehicle. If the presence of the obstacle is validated, the avoidance algorithm sends information of reference to the trajectory control [5] [6] [7] [8].

Deliberative architecture is based on a structure of flow of sensor, plan and action. This architecture can be used, if the map of environment of navigation with the obstacles present is known, and if the vehicle has the ability to locate itself within that map. Its disadvantage is a high computational cost due to the use of type algorithms to Wavefront, Potential field, $\mathrm{A}^{*}$ and Genetic algorithms, among others.

Finally, there is the hybrid architecture. This method combines the advantages and disadvantages of the above strategies, as a short reaction time in presence of sudden obstacles, robustness, high computational cost and an optimal trajectory of navigation. however, as the previous architecture, it needs the map of the environment of navigation.

For our case study, we generated a list of requirements to be met by the obstacles avoidance system (anti-collision system) to be used, based on the current needs of AUV, which are:

- Short reaction time in the presence of obstacles.

- Low computational costs. Compatible with vehicle unit control (PC / 104- plus (AMD® LX800), Ubuntu and LabView).

- $\quad$ No need for a map of navigation environment.

- Maneuvers suited to the dimensions, navigation speed (actual forward speed of the AUV) and turning radius of the vehicle.

- Capacity of obstacle avoidance static and in moving.

- It can be programmed to accomplish with the COLREGS [9] regulations.

When analyzing the characteristics of each architectures and having clear the requirements that the CAS must comply, it has been selected reactive architecture.

This provides greater flexibility, shorter reaction time, robustness against sudden obstacles and low computational cost.
Therefore, the motion or navigation planning of the vehicle Guanay II, is designed combining the strategies of trajectory control based in follow-up of waypoint (Purepursuit and Path Following) with a strategy of obstacle avoidance based on a reactive architecture.

This combination allows the vehicle to navigate between waypoints securely. The trajectory control (Pure-pursuit or Path Following) generate the benchmarks of the inner loop corresponding to the yaw angle and forward speed, but in the presence of an obstacle these benchmarks will be generated by the obstacles avoidance system.

As a contribution obstacle avoidance system with a reactive architecture implemented through by a fuzzy system is designed. The inference of the system will depend on vehicle speed (high or low), the distance to the obstacle and the location of the obstacle on the trajectory presented at IV.B. The limits of the membership functions input and output of the system will depend on the vehicle speed presented at IV.A, this will allow greater maneuverability at low speed.

\section{Design Of Obstacle Avoidance System}

Between several possibilities for implementing a reactive architecture, we find that the appropriate option in our case is the use of a fuzzy system [7] [8] [10] [11], which has been described by some authors as a similar system as the expert systems, since it may represent the knowledge in a set of rules of conditional structure. This allows the implementation of the navigation rules not only commensurate with the rules COLREGS, but also commensurate with the physical and operational characteristics of the vehicle.

Another advantage of the utilization of a fuzzy system focuses on system response to uncertainty and management of the real information, that can include noise and errors.

By definition fuzzy systems [12] are divided into 7 stages: definition of input and output variables, allocation of memberships functions, fuzzification, rule evaluation, inference, aggregate and defuzzyfication. The common case that is found in the literature, is the use of a single fuzzy control, to perform the task of obstacle avoidance [7] [10].

The fuzzification corresponds to quantifying the value of the input variable with respect to the membership functions. The evaluation of the rules and inference, is based on a conditional equation of the type "IF...THEN...", defining the control strategy and determining the output set for each of the rules. The aggregate and defuzzification allow to obtain the most representative value of the output variable from an operation of all sets of output.

The obstacle avoidance system has been designed according to the block diagram shown in Fig. 4. The system has four input variables that are navigation speed (the actual forward speed of the AUV) and distance to the obstacle in the sectors: center, right and left, as defined in Fig. 3. The output variables are the new references of the forward speed and the yaw angle (input to inner loop). 


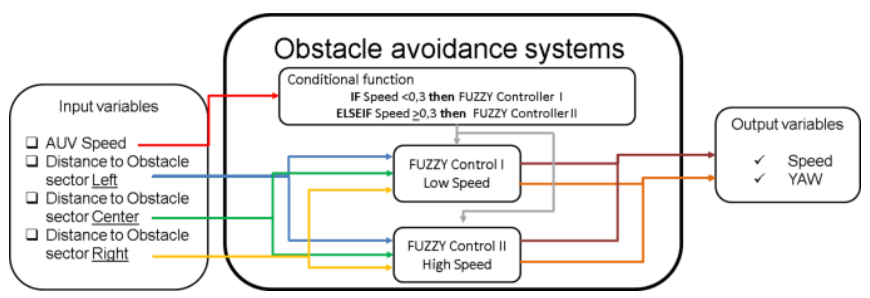

Figure 4. Block diagram of obstacle avoidance system

The input variable of the "navigation speed" is used to control a conditional function that selects one of the two fuzzy controllers implemented. Each controllers responds at a "navigation speed" of the vehicle because the turning radius is highly dependent on speed, as we have seen in Fig.2. If the navigation speed is less than $0.3 \mathrm{~m} / \mathrm{s}$ acts the fuzzy controller I and if it is greater or equal acts the fuzzy controller II.

The characteristics of fuzzy control are detailed below.

\section{A. Inputs variables and Outputs variables}

Each of the fuzzy controllers has three input variables that indicate the distance to the obstacle in the sectors (Fig.3): center, right and left of the route, are called distance right sector (DR) distance center sector (DC) and distance left sector (DL).

For each of these input variables, have been defined three membership functions, type: trapezoidal, triangular and trapezoidal, called respectively as: high, medium and low risk of collision. (Fig.5).

The limits of the memberships functions have been defined regarding to the maximum speed of the vehicle (1 $\mathrm{m} / \mathrm{s}$ ), the runtime of the obstacle detection software, the turning radius and the distance traveled by the vehicle during runtime the obstacle detection algorithm.
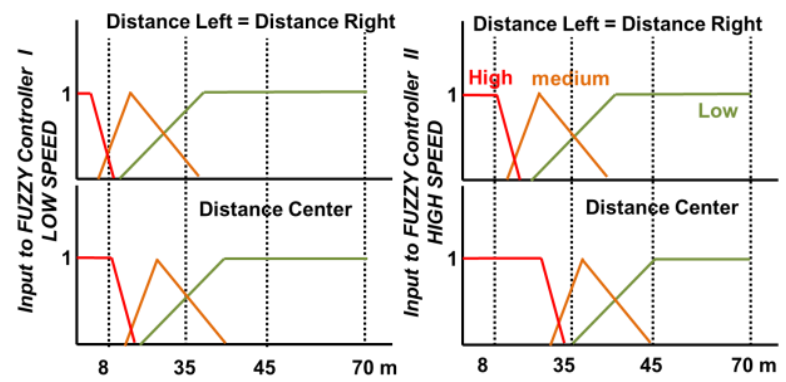

Figure 5. Diagram of input variables and the membership functions

Each of these fuzzy controllers also have two output variables corresponding to "reference yaw" and the "reference speed". To the output variable "reference yaw" are assigned 6 memberships functions of the type triangular. For the fuzzy controller II, which applies to high-speed of navigation, the output range is between $+/-90^{\circ}$, while for the fuzzy controller I, that applies for low speed, the output range is $+/-180^{\circ}$.

To the variable of "reference speed" are assigned three membership functions of type: triangular and trapezoidal in a range of $0 \mathrm{~m} / \mathrm{s}$ to $1 \mathrm{~m} / \mathrm{s}$. The characteristics of output variables can be seen in Fig.6.
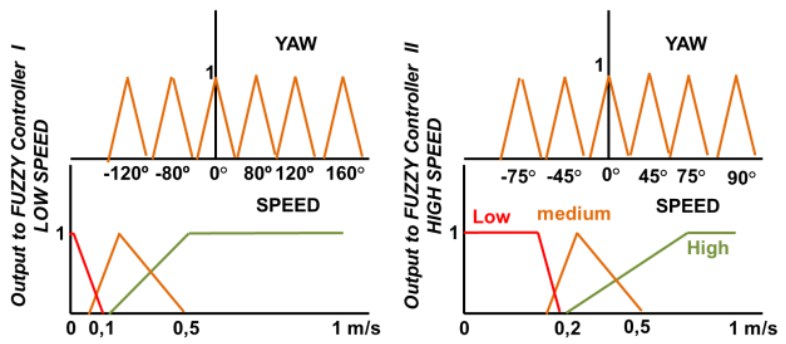

Figure 6. Diagram of output variables and the membership functions

\section{B. Rule-based and inference}

The rule base and inference, allows calculating the membership function of the output variable as from the combination of the input variables for each of the rules that form the basis of rules.

In this proposal the rule base has been built to guarantee the safety of the vehicle and comply with the COLREGS [9] regulations. This normative indicating that before the presence of an obstacle the vehicle must, as far as possible, drive on the right hand side at low speed, without jeopardizing its own security, in the event of compromise their safety, its free to take countermeasures such as turn left, having as reference vehicle maneuverability, braking distance, turning radius. It also recommends that all the maneuvers if circumstances allow should be made clearly and in sufficient time, in compliance with the good practices. The rule-base implemented in both controllers is composed of 27 rules, although each controller in the consequents has different values in the output variable of the yaw. In Table I, the two sets of rules are defined, indicating on the column corresponding to the output variable yaw, the values of fuzzy control I and the fuzzy control II. The conditional equation used is kind Mamdani [12] and corresponds to:

$$
\begin{aligned}
& \text { If Left }=\ldots \text { AND Center }=\ldots \text { AND Right }=\ldots \text { Then } \text { Speed }=\ldots \\
& \qquad \text { AND Yaw }=\ldots
\end{aligned}
$$

TABLE I. RULES

\begin{tabular}{|c|c|c|c|c|c|}
\hline & \multicolumn{3}{|c|}{ Input variables } & \multicolumn{2}{c|}{ Output variables } \\
\hline$\#$ & Left & Center & Right & Speed & Yaw (H/L) \\
\hline 1 & High & High & High & Low & $90^{\circ} / 160^{\circ}$ \\
\hline 2 & High & High & medium & Low & $75^{\circ} / 120^{\circ}$ \\
\hline 3 & High & High & Low & Low & $45^{\circ} / 80^{\circ}$ \\
\hline 4 & High & medium & High & Low & $45^{\circ} / 80^{\circ}$ \\
\hline 5 & High & medium & medium & Low & $45^{\circ} / 80^{\circ}$ \\
\hline 6 & High & medium & Low & medium & $45^{\circ} / 80^{\circ}$ \\
\hline 7 & High & Low & High & Low & $0^{\circ}$ \\
\hline 8 & High & Low & medium & medium & $0^{\circ}$ \\
\hline 9 & High & Low & Low & medium & $0^{\circ}$ \\
\hline 10 & medium & High & High & Low & $-75^{\circ} /-120^{\circ}$ \\
\hline 11 & medium & High & medium & Low & $45^{\circ} / 80^{\circ}$ \\
\hline 12 & medium & High & Low & Low & $45^{\circ} / 80^{\circ}$ \\
\hline 13 & medium & medium & High & Low & $-75^{\circ} /-120^{\circ}$ \\
\hline 14 & medium & medium & medium & Low & $45^{\circ} / 80^{\circ}$ \\
\hline 15 & medium & medium & Low & medium & $45^{\circ} / 80^{\circ}$ \\
\hline 16 & medium & Low & High & Low & $0^{\circ}$ \\
\hline 17 & medium & Low & medium & Low & $0^{\circ}$ \\
\hline
\end{tabular}




\begin{tabular}{|c|c|c|c|c|c|}
\hline 18 & medium & Low & Low & High & $0^{\circ}$ \\
\hline 19 & Low & High & High & Low & $-45^{\circ} /-80^{\circ}$ \\
\hline 20 & Low & High & medium & Low & $-45^{\circ} /-80^{\circ}$ \\
\hline 21 & Low & High & Low & Low & $45^{\circ} / 80^{\circ}$ \\
\hline 22 & Low & medium & High & medium & $-45^{\circ} /-80^{\circ}$ \\
\hline 23 & Low & medium & medium & Low & $-45^{\circ} /-80^{\circ}$ \\
\hline 24 & Low & medium & Low & Low & $45^{\circ} / 80^{\circ}$ \\
\hline 25 & Low & Low & High & medium & $0^{\circ}$ \\
\hline 26 & Low & Low & medium & High & $0^{\circ}$ \\
\hline 27 & Low & Low & Low & High & $0^{\circ}$ \\
\hline
\end{tabular}

\section{Aggregation and, defuzzification}

The evaluation of the rule base (1), is carried out using the inference of Mamdani, with an aggregate function type max and a defuzzification by the centroid method. [12]

\section{Simulation EnVironment Of Obstacle Avoidance System}

In previous works, we have developed algorithms for the simulation of vehicle Guanay II, in three degrees of freedom [2], and the obstacle detection system [3], their characteristics are summarized in Table II.

For the development of the simulations, the obstacle avoidance system proposed in this paper has been implemented in a Matlab script. The two fuzzy controls designed and described in this research have been implemented using the Fuzzy Logic Toolbox.

TABLE II. SIMULATION PARAMETERS

\begin{tabular}{|l|c|}
\hline \multicolumn{1}{|c|}{ Parameters } & Value \\
\hline Mechanical operating range sonar & $90^{\circ}$ \\
\hline turning mechanical resolution sonar & $0.9^{\circ}$ \\
\hline Number of measuring points sonar & 400 \\
\hline Maximum operating distance sonar & $70 \mathrm{~m}$ \\
\hline Image resolution & $1 \mathrm{~m}$ \\
\hline Radius waypoint & $5 \mathrm{~m}$ \\
\hline Speed AUV & Variable $(0-1 \mathrm{~m} / \mathrm{s})$ \\
\hline Path Control & Pure-pursuit \\
\hline Obstacle avoidance system & Reactive-Fuzzy \\
\hline
\end{tabular}

\section{SimUlation AND ANALYSIS}

In order to validate the correct operation of avoidance algorithm designed, we have simulated two route composed of a total of eight waypoints strategically located. In the first route, the first five waypoints allow to evaluate the behavior of AUV in a complex environment, where the vehicle moves through a narrow path, and frontally finds different obstacles (circles of radius 5 and $10 \mathrm{~m}$ ) (Fig.7).

In the second route, through the waypoints 6,7 and 8 , a specific case is evaluated, which is the navigation into a corner. The waypoint 7 forces the AUV to enter in this area, which it's considered as a difficult place for navigation. On the other hand, the waypoint 8 is not positioned in direct line of sight. Therefore, the vehicle must face an obstacle in the form of L (death point), which represents a high risk of collision (Fig.8).

When analyzing the two routes simulated, we can see that it has given the Guanay II a system of safe navigation around obstacles without losing the trajectory of navigation and reaching each of the waypoints. The vehicle during the route
1 remains at a minimum distance of 5 meters of the obstacles, reducing its references forward speed of $1 \mathrm{~m} / \mathrm{s}$ to values between $0.02 \mathrm{~m} / \mathrm{s}$ and $0.4 \mathrm{~m} / \mathrm{s}$.

During the route 2, where a deadlock situation occurs, the distance between the vehicle and certain sections of the obstacle in the form of $\mathrm{L}$ are 1 to 2 meters, but the vehicle will always be sailing at very low speed; less than $0,1 \mathrm{~m} / \mathrm{s}$. The maneuvers performed by the vehicle to go out the deadlock and reach the waypoint 8 is based on making small movements at low speed and with a changing in the yaw with an approximate value of $160^{\circ}$, relative to the current direction of the vehicle. With this maneuver, the vehicle turns in a small area until it finds a perpendicular route free of obstacles, and at that moment, the trajectory control will correct the direction of the vehicle, by taking it to the next waypoint.

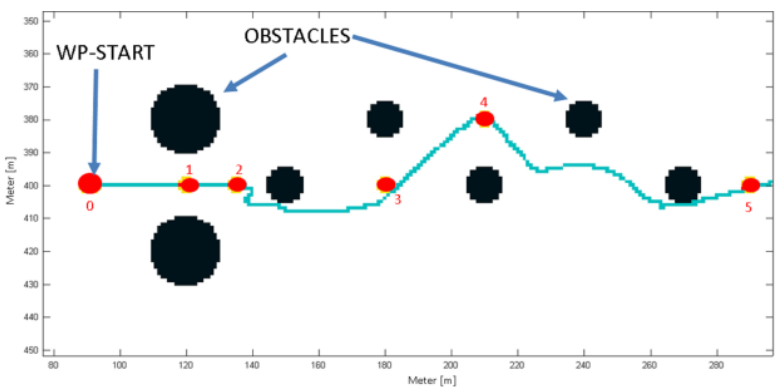

Figure 7. Simulation navigation route 1

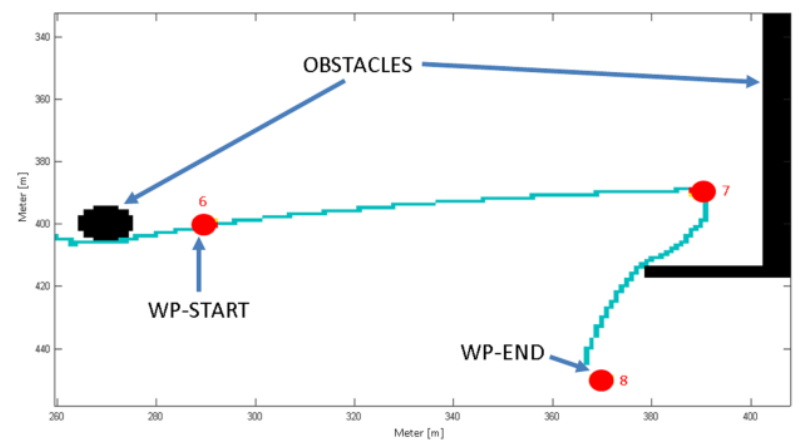

Figure 8. Simulation navigation route 2

\section{CONCLUSIONS}

Different simulations have been performed with different obstacles, changing its location and shape. We have different waypoints strategically located, that allow us to study and analyze the behavior of the navigation system, composed of the trajectory control and obstacle avoidance system. The results obtained have been shown in Fig.7 and 8. When analyzing these images, we observe that the vehicle evades different obstacles, while performing correct navigation. These simulations confirmed the proper functioning of the system, achieving detection, avoidance of obstacles and navigation in confined spaces. Having validated the functioning of the system designed, it's now possible to introduce the algorithms into the unit control of the Guanay II AUV, for perform tests. 


\section{ACKNOWLEDGMENT}

We acknowledge the financial support from Spanish Ministerio de Economia y Competitividad under contract CGL2013- 42557-R (Interoperability and implementation of autonomous marine platforms for seismic monitoring INTMARSIS) and the project NeXOS from the European Union's Seventh Programme for research, technological development and demonstration under grant agreement No 614102 .

\section{REFERENCES}

[1] Gomariz, S.; Masmitja, I.; González, J.; Masmitjà, G.; Prats, J. "Guanay-II: an autonomous underwater vehicle for vertical/horizontal sampling". Journal of marine science and technology. 2014. ISSN: 0948-4280. DOI 10.1007/s00773-013-0253-y. URL: http://link.springer.com/article/10.1007\%2Fs00773-013-0253-y

[2] González, J; Thesis Contribution to the Model and Navigation Control of an Autonomous Underwater Vehicle, Universidad Politécnica de Catalunya, 2015, URL: http://www.tdx.cat/handle/10803/311422

[3] Galarza, C., Masmitja, I., González, J., Prat, J., Gomariz, S., Del Rio, J. Design obstacle detection system for AUV Guanay II. A: International Workshop on Marine Technology. "MARTECH 2015 : Sixth International Workshop on Marine Technology : Cartagena, September 15th, 16th and 17th". Cartagena: 2015, p. 15-18. URL: http://hdl.handle.net/2117/77240 ISBN: 978-84-608-1708-6

[4] Tritech, Micron Sonar, Document: 0650-SOM-00004, Issue: 02, URL: http://www.tritech.co.uk/media/products/small-rov-mechanicalsector-scanning-sonar-tritech-micron.pdf

[5] Øystein Engelhardtsen, 3D AUV Collision Avoidance, Norwegian University of Science and Technology ,Department of Engineering Cybernetics,2007, Accessed November 30, 2014. URL: http://daim.idi.ntnu.no/masteroppgaver/003/3362/tittelside.pdf

[6] M.L. Seto, L. Paull, and S. Saeedi, MARINE ROBOT AUTONOMY, ISBN 9781461456599, 9781461456582, 2013. URL: http://www.springer.com/us/book/9781461456582

[7] Hromatka, Michelle, "A Fuzzy Logic Approach to Collision Avoidance in Smart UAVs" (2013). Honors Theses. Paper 13. URL: http://digitalcommons.csbsju.edu/honors_theses/13

[8] J.Evans, P.Patrón, B.Smith. D.M. Lane. "Design and evaluation of a reactive and deliberative collision avoidance and escape architecture for autonomous robots" Autonomous Robots Volume 24, Number 3 (2008), 247-266 URL: http://link.springer.com/article/10.1007\%2Fs10514-007-9053-8

[9] Lloyd's Register or International Maritime Organization, COLREGS International Regulations for Preventing Collisions at Sea 2005, Accessed January 20, 2015. URL: http://www.mar.ist.utl.pt/mventura/Projecto-Navios-I/IMOConventions\%20(copies)/COLREG-1972.pdf

[10] TAN, CHIEW SEON, Thesis A COLLISION AVOIDANCE SYSTEM FOR AUTONOMOUS UNDERWATER VEHICLES, 2006, University of Plymouth, Faculty of Science and Technology, URL: $\underline{\text { http://hdl.handle.net/10026.1/2258 }}$

[11] Shifei Liu, Yanhui Wei ; Yanbin Gao, 3D path planning for AUV using fuzzy logic, Computer Science and Information Processing (CSIP), 2012 International Conference on, 24-26 Aug. 2012, IEEE, DOI: $\quad 10.1109 /$ CSIP.2012.6308925, URL: http://ieeexplore.ieee.org/stamp/stamp.jsp?tp=\&arnumber=6308925

[12] Hao Ying, Fuzzy Control and Modeling:Analytical Foundations and Applications, Wiley-IEEE Press, 2000, ISBN : 9780470544730, URL: $\mathrm{http}$ ///ieeexplore.ieee.org/servlet/opac?bknumber $=5263877$ 